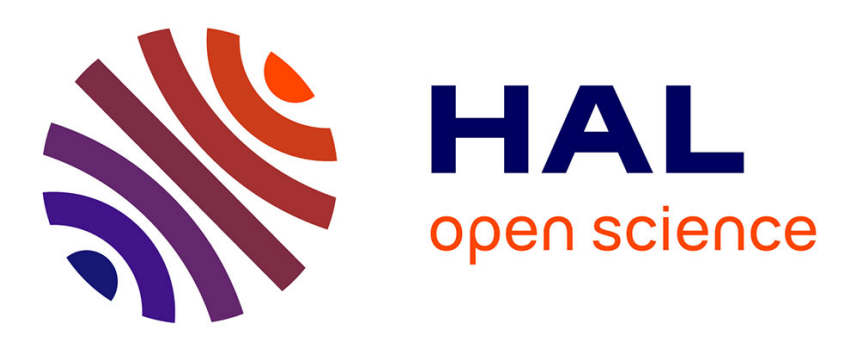

\title{
A Broadband Electromagnetic Homogenization Method for Composite Materials
}

Ghida Al Achkar, Lionel Pichon, Nabil Benjelloun, Laurent Daniel

\section{To cite this version:}

Ghida Al Achkar, Lionel Pichon, Nabil Benjelloun, Laurent Daniel. A Broadband Electromagnetic Homogenization Method for Composite Materials. IEEE Transactions on Magnetics, 2018, 54 (3), pp.8080147. 10.1109/TMAG.2017.2759224 . hal-01800705

\section{HAL Id: hal-01800705 https://hal.science/hal-01800705}

Submitted on 28 Jul 2020

HAL is a multi-disciplinary open access archive for the deposit and dissemination of scientific research documents, whether they are published or not. The documents may come from teaching and research institutions in France or abroad, or from public or private research centers.
L'archive ouverte pluridisciplinaire HAL, est destinée au dépôt et à la diffusion de documents scientifiques de niveau recherche, publiés ou non, émanant des établissements d'enseignement et de recherche français ou étrangers, des laboratoires publics ou privés. 


\title{
A broadband electromagnetic homogenization method for composite materials
}

\author{
Ghida Al Achkar ${ }^{1,2}$, student member, IEEE, Lionel Pichon ${ }^{1}$, Nabil Benjelloun ${ }^{2}$, and Laurent Daniel ${ }^{1}$ \\ ${ }^{1}$ GeePs | Group of electrical engineering - Paris, CNRS (UMR 8507) / CentraleSupélec / UPMC / Univ Paris-Sud / \\ Univ. Paris-Saclay, 91192 Gif sur Yvette, France \\ ${ }^{2}$ IRSEEM-ESIGELEC-EA 4353, av. Galilée, 76801 St-Et-du-Rouvray, France
}

\begin{abstract}
This paper focuses on broadband electromagnetic (EM) modeling of composite materials. A homogenization method is carried out by combining finite element calculations with a numerical inversion technique: first, the transmission and reflection coefficients of the composite plate are computed over a large frequency band (up to $50 \mathrm{GHz}$ ) using a 3D finite element method. Then an inversion technique is applied in order to extract electromagnetic properties. This approach extends the application domain of classical homogenization methods by enabling the modeling of different types of composites.
\end{abstract}

Index Terms - 3D Finite Element Method (FEM), Composite materials, Electromagnetic properties, Homogenization.

\section{INTRODUCTION}

$\mathrm{T}$ HE automotive industry is increasingly replacing metal alloys with composite materials due to their appealing mechanical properties. These structures offer similar robustness while having a significantly lighter weight. In order to benefit from these characteristics for EM shielding applications, e.g. isolate vehicle electronics from external waves, proper models defining the behavior of such materials should be established. However, because composites are essentially heterogeneous materials, there exists a contrast in EM properties between the matrix (that holds the material together) and the inclusion particles (fibers, ellipsoids, spheres, etc.) that constitute them. Each phase has a different EM response and makes the composite's general behavior complicated to model. This explains the need for homogenization methods that can describe their overall electromagnetic response, by defining a - fictitious homogeneous medium that shows an identical behavior when illuminated by an EM wave at a given frequency.

Several analytical mixing rules have been developed (a comparative study is presented in [1]), but their application domain is restricted to static excitations and low fiber volume ratio. While the Maxwell-Garnett method can be extended for biphasic materials when the inclusion size is smaller than the incident wavelength, it still does not provide an accurate estimation of their effective properties at relatively high frequencies. As for woven fiber reinforcements, the work in literature has relied on the periodicity that they present in order to use numerical method and therefore extract the material's shielding efficiency as done in [2] using a rigorous coupled wave analysis technique. Moreover, in [3] and [4], multiple experimental methods are used to measure the $\mathrm{S}$

Manuscript received June 26, 2017; revised August 28, 2017; accepted

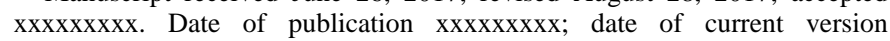
xxxxxxxxx. Corresponding author: Ghida Al Achkar (e-mail: ghida.alachkar@centralesupelec.fr).

Color versions of one or more of the figures in this paper are available online at http://ieeexplore.ieee.org.

Digital Object Identifier (inserted by IEEE). parameters of a composite plate which can lead to explain the overall behavior of the material.

In this paper, we focus on composites made from conductive fibers and dielectric matrix. The homogenization method we present can be used to model various types of inclusions, woven fibers in particular, by combining a finite element (FEM) approach with an inversion technique. First, the theory behind this work is presented: the model used for the simulations is described as well as the adopted inversion method. Then, the implementation results are analyzed and a conclusion is drawn.

\section{HOMOGENIZATION METHOD}

\section{A. 3D FEM Model}

The first step to calculating the effective EM properties is to get the transmission coefficient of the composite plate in the frequency band of interest.

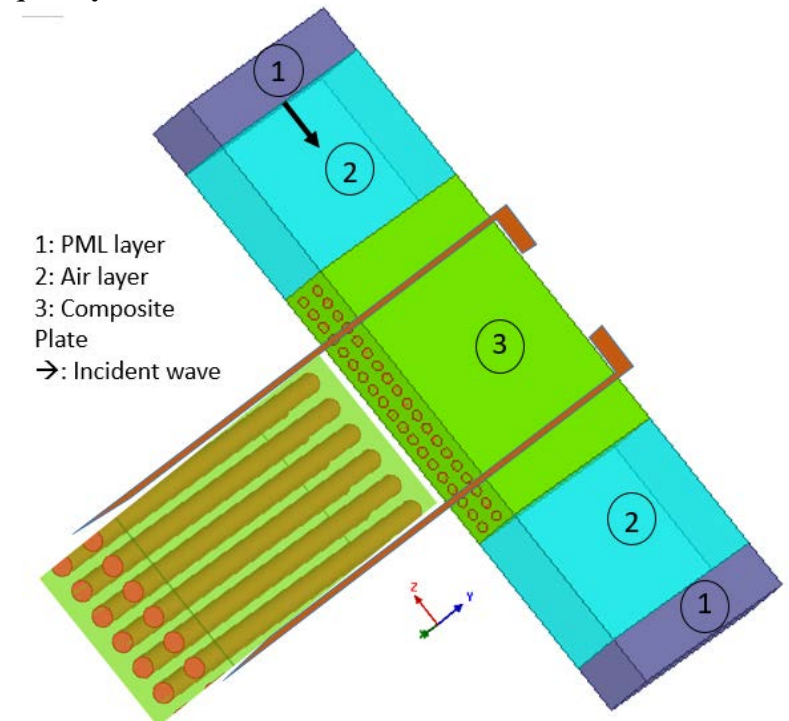

Fig. 1. 3D FEM model: Composite plate with cylindrical inclusions

One of the main goals is to consider different shapes of inclusions, so a finite element software was adopted as a simulation tool. The 2D model described in [5] to calculate the shielding effectiveness of composites is expanded into a $3 \mathrm{D}$ 
model. A composite slab located in air is illuminated with an electric field of normal incidence. Fig. 1 shows the computational domain with cylindrical inclusions that can be replaced with any other geometry, thus making this model an adaptable one.

In the interest of accurately modeling the structure, the chosen volume element should be large enough to represent its overall response while maintaining a reasonable simulation time. The composite plate is surrounded by air. Neumann boundary conditions are applied to the appropriate surfaces, and Perfectly Matched Layers (PMLs) are added to simulate infinite domains. Maxwell's equations are solved in frequency domain for the electric field: $\vec{E}(x, y, z)$. In our case we treat materials of a negligible absorption ratio. This means that all the information about their properties is fully conveyed by either the transmission or the reflection coefficient. Therefore using any of them should lead to the same results. Here we choose to work with the former. The shielding effectiveness $S E$ is then extracted by calculating the S-parameters of the composite plate (1).

$$
S E_{S I M(d B)}=-S_{21(d B)}=20 . \log _{10} \frac{\left|\vec{E}_{\text {incident }}\right|}{\left|\vec{E}_{\text {transmitted }}\right|}
$$

\section{B. Retrieving EM properties from S-parameters}

Having numerically calculated the shielding effectiveness of our heterogeneous medium, the aim now is to define the EM properties of a homogeneous plate that would have an identical response. To do so, we first define the theoretical $S E_{\text {theo. }}$ of such homogeneous plate as a function of its thickness $l$, the propagation constant $k$, the refractive index $n$, as well as its properties: permittivity $\varepsilon$ and permeability $\mu$ (2)(3). Such expressions are defined for a normally incident EM wave. This assumption will be used throughout the paper. However, treating a case of oblique incidence is possible, though requires defining the transmittance as a function of the angle of incidence, the wave polarization, the complex material impedance, etc. (Fresnel's equations). In practical applications involving shielding boxes, the orientation of the incident wave relative to the different walls of the 3D structure is not known. Considering the normal incidence case is an approximation which is generally sufficient to obtain the magnitude of the shielding effectiveness of the enclosure.

$$
\begin{gathered}
S E_{\text {theo. }}=e^{j k l}\left(1-q e^{-2 j k l}\right) / p \\
q=\left(\frac{n / \mu_{r}-1}{n / \mu_{r}+1}\right)^{2} \text { and } p=\frac{4 n / \mu_{r}}{\left(n / \mu_{r}+1\right)^{2}} \\
F_{c}=\left|S E_{S I M}-S E_{\text {theo. }}\left(\varepsilon^{*}, \mu^{*}\right)\right| \\
=\left|S E_{S I M}-e^{j l \sqrt{\varepsilon_{0} \cdot \mu_{0} \cdot \omega^{2}-j \mu_{0} \sigma \cdot \omega}}\left(1-q e^{-j 2 l \sqrt{\varepsilon_{0} \cdot \mu_{0} \cdot \omega^{2}-j \mu_{0} \sigma . \omega}}\right) / p\right|
\end{gathered}
$$

The sought effective properties are the solution to an optimization problem, because they bring to a minimum the distance between the SE of the composite plate and that of an equivalent homogeneous one of the same thickness (4).
In the following, we are interested in composites exhibiting a high contrast in conductivity, such as dielectric matrices and carbon based fibers, thus limiting the cost function $F_{c}$ to one effective property. The optimization method must be adapted to the cost function at hand. Since its variations might be highly nonlinear at certain intervals and the presence of multiple local minima is not eliminated, genetic algorithms GAs are known to best handle these types of conditions for minimization problems. However, while they offer the above listed advantages, their convergence is generally slow around the global optimum [6]. This leads to the combination of a deterministic algorithm with the GA in order to achieve a faster, more accurate convergence once in the neighborhood of the global minimum. The GA is based on an iterative process, a detailed explanation of which can be found in [7]. Once a stopping criterion is reached, the estimated intermediate solution is considered to be a starting point for the quasiNewton method, which will compute the final value of the effective EM property. The values for the different parameters used in these algorithms are described in III.

\section{MODELING RESULTS}

\section{A. Composite plate with unidirectional cylindrical inclusions}

The FEM model detailed in II-A was applied to a $l=6 \mathrm{~mm}$ thick composite plate with 30 rows of cylindrical inclusions having a $25 \mu \mathrm{m}$ radius each and occupying a volume fraction of $f_{v}=19.63 \%$. The geometry is meshed using triangular prismatic elements for the inclusions and regular tetrahedral elements for the surroundings. A contrast in conductivity between the matrix and the reinforcement is considered, Table I specifies the EM properties of each phase.

TABLE I : EM PROPERTIES OF THE COMPOSITE PlATE

\begin{tabular}{lccc}
\hline \hline Phase & $\begin{array}{c}\text { Relative } \\
\text { Permittivity }\end{array}$ & $\begin{array}{c}\text { Conductivity } \\
\sigma[\mathrm{S} / \mathrm{m}]\end{array}$ & $\begin{array}{c}\text { Relative } \\
\text { Permeability } \mu_{\mathrm{r}}\end{array}$ \\
\hline Matrix & $\varepsilon_{m}=1$ & $\sigma_{m}=0$ & 1 \\
Inclusions & $\varepsilon_{f}=1$ & $\sigma_{f}=\left\{10^{3} ; 4.10^{3} ; 10^{4}\right\}$ & 1 \\
\hline \hline
\end{tabular}

The incident wave is propagating along the $\mathrm{z}$-axis perpendicularly to the fibers, according to (5) where $\mathrm{k}_{0}$ is the propagation constant and $\mathrm{u}_{\mathrm{x}}$ is the unit vector of the $\mathrm{x}$-axis.

$$
E_{\text {inc }}=E \cdot e^{j\left(\omega t-k_{0} z\right)} u_{x}
$$

The inversion technique described in II-B is now used to compute the effective conductivity of the plate. At first the GA computes an initial estimate using a population size of 50 candidates at each iteration. The crossover probability is set to 0.8 and the mutation rate decreases as the algorithm approaches the solution. The algorithm stops once the difference between the present best fitness function value and the previous one becomes less than 0.2. The quasi-Newton algorithm then takes over and computes the minimum of the cost function to a precision of $10^{-6}$. Fig. 3 shows the results which demonstrate a behavior similar to that of the shielding efficiency in Fig. 2. 


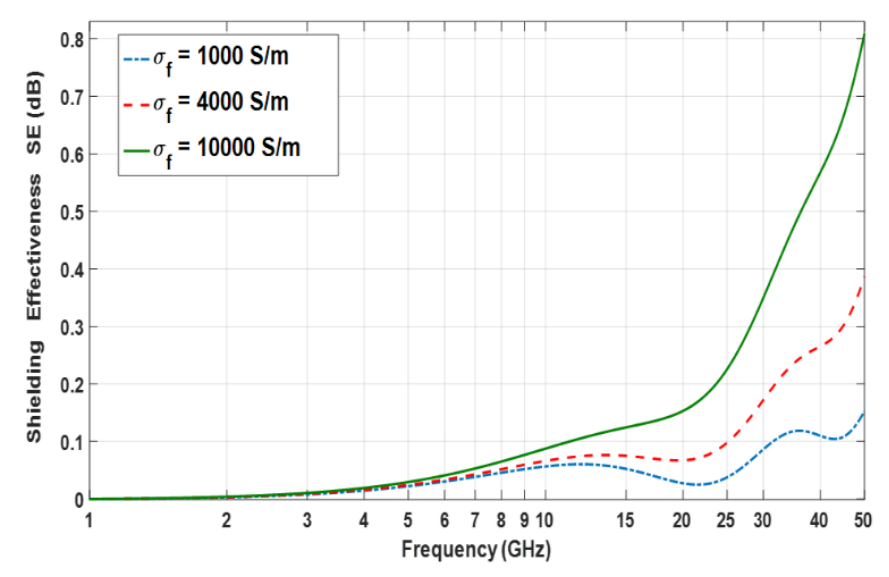

Fig. 2. Transmission coefficient (in $\mathrm{dB}$ ) of the composite plate for different fiber conductivities as a function of the frequency (in $\mathrm{GHz}$ )

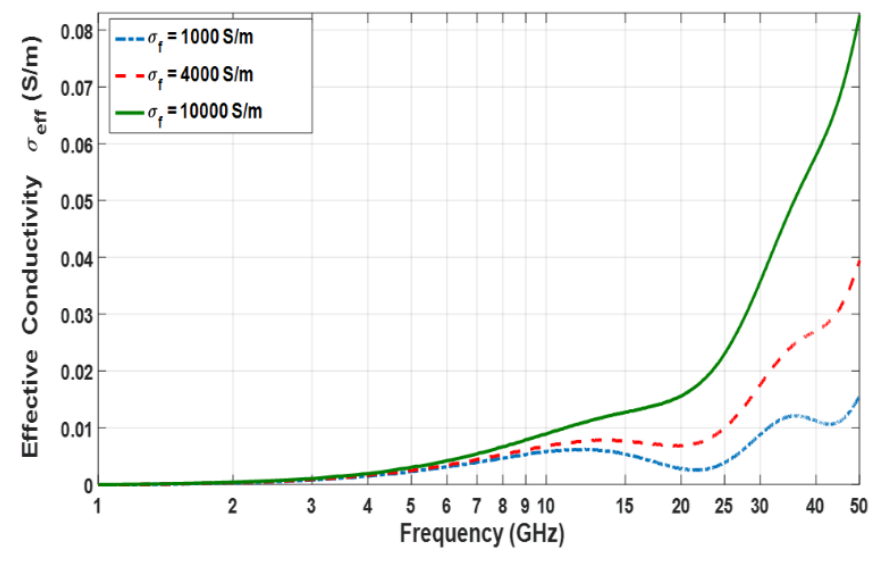

Fig. 3. Effective conductivity of the plate vs. frequency

The low inclusion volume percentage considered in this example model makes it possible to conduct a comparative study in order to place this technique with respect to the classical mixing rules, as well as other homogenization methods. Two models are considered:

- The formula developed by Maxwell-Garnett MG and applied mostly to biphasic materials in the case of quasistatic excitations (6).

$$
\varepsilon_{M G}^{*}=\varepsilon_{m}^{*}+\frac{3 \cdot f_{v} \cdot \varepsilon_{m}^{*} \frac{\varepsilon_{m}^{*}-\varepsilon_{f}^{*}}{\varepsilon_{m}^{*}+2 \varepsilon_{f}^{*}}}{1-f_{v} \frac{\varepsilon_{m}^{*}-\varepsilon_{f}^{*}}{\varepsilon_{m}^{*}+2 \varepsilon_{f}^{*}}}
$$

- The dynamic homogenization method DHM developed in [5] and adapted to model composites at high frequencies by introducing an inclusion size/ wavelength ratio into the calculation of effective properties thus accounting for the interaction between the incident wave and the material.

The results for $\sigma_{m}=0 \mathrm{~S} / \mathrm{m}$ and $\sigma_{f}=10^{4} \mathrm{~S} / \mathrm{m}$ (Fig. 4) show that the MG model is not convenient for microwave frequencies, because it underestimates the effective conductivity of the composite.
As to the estimate used in [5], and detailed in Appendix A, it estimates the effective conductivity to be higher than the values we get with this technique. The reason behind these differences is that the DHM calculates a complex permittivity and therefore an effective permittivity as well as conductivity for a contrast in any of these two properties, thus giving the same SE as the one calculated using a FEM. Whereas in our case we consider that the effective permittivity is constant and proceed to estimate the conductivity of the homogenized plate.

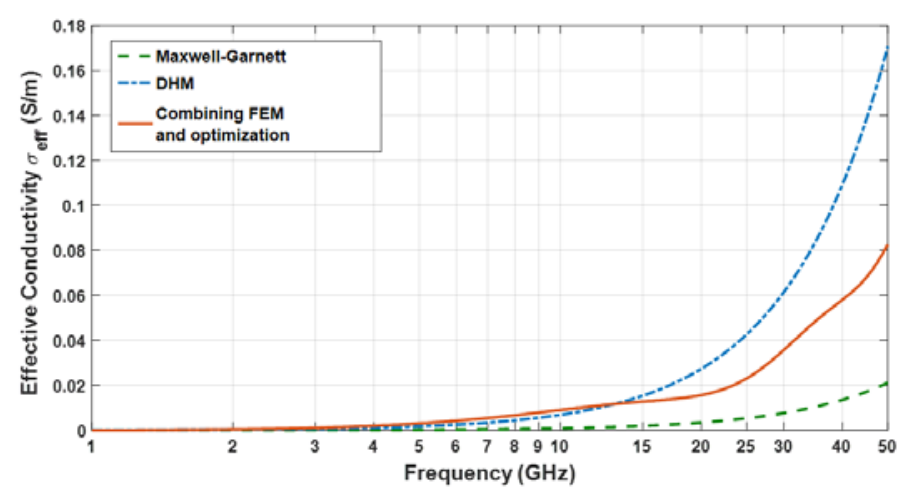

Fig. 4. Comparative study of three different homogenization techniques

\section{B. Electromagnetic behavior of multilayered composites}

In this part, we investigate the shielding effectiveness of a composite plate having two consecutive layers of unidirectional fibers distributed in perpendicular directions (Fig.5). The same FEM model is applied to each layer and a symmetrical S-parameter matrix is derived from the calculations. A global shielding coefficient is then derived by cascading the transmission matrices (computed from the scattering parameters as demonstrated in [8]).

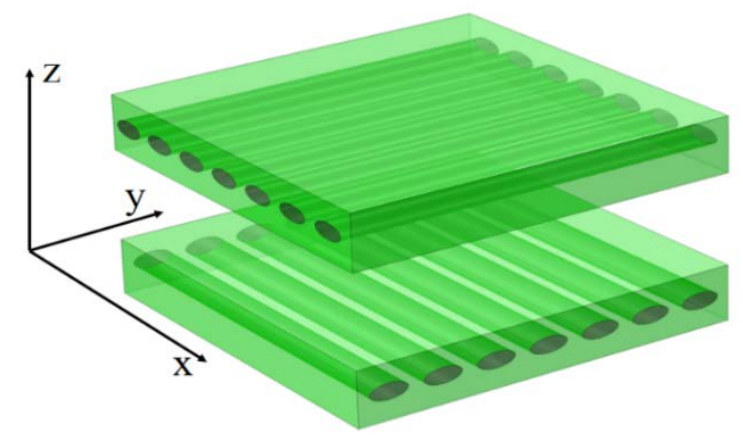

Fig. 5. Two layer composite material made from unidirectional fibers (with elliptical cross sections) in opposite directions.

This model gives an idea of the shielding effectiveness' order of magnitude for woven composites with $90^{\circ}$ weaving angles. The incident electric wave is x-polarized (5) and the different geometrical dimensions and EM properties are detailed in Table II. Fig. 6 shows the shielding effectiveness for each of the layers separately as well as the global SE (for a fiber conductivity $\sigma_{f}=10^{3} \mathrm{~S} / \mathrm{m}$ ).

The major part of the shielding seems to be ensured by the fibers that are parallel to the polarization of the electric field. As for the oscillations at high frequencies, the figure shows that they don't follow the same pattern as the transmission coefficient $S_{21}$. 
TABLE II : EM PRoperties AND DimENSIONS OF THE COMPOSITE PlATE

\begin{tabular}{lcc}
\hline \hline EM Property / Dimension & Symbol (Unit) & Value \\
\hline Relative permittivity & $\varepsilon_{m}, \varepsilon_{f}$ & 1 \\
Relative permeability & $\mu_{m}, \mu_{f}$ & 1 \\
Matrix conductivity & $\sigma_{m}(S / m)$ & 0 \\
Fiber conductivity & $\sigma_{f}(S / m)$ & $\left\{5.10^{2} ; 10^{3}\right\}$ \\
Height (for the entire plate) & $l_{z}(\mathrm{~mm})$ & 3 \\
Semi-major axis & $b(\mathrm{~mm})$ & 0.625 \\
Semi-minor axis & $a(\mathrm{~mm})$ & 0.25 \\
Fiber volume ratio & $f_{v}(\%)$ & 14 \\
\hline \hline
\end{tabular}

This is because they are also influenced by the reflection parameters $S_{11}$ and $S_{22}$. These coefficients are integrated within the calculation of the global $S E$ through the scattering matrices and show a higher level of oscillation, thus explaining the variations represented here.

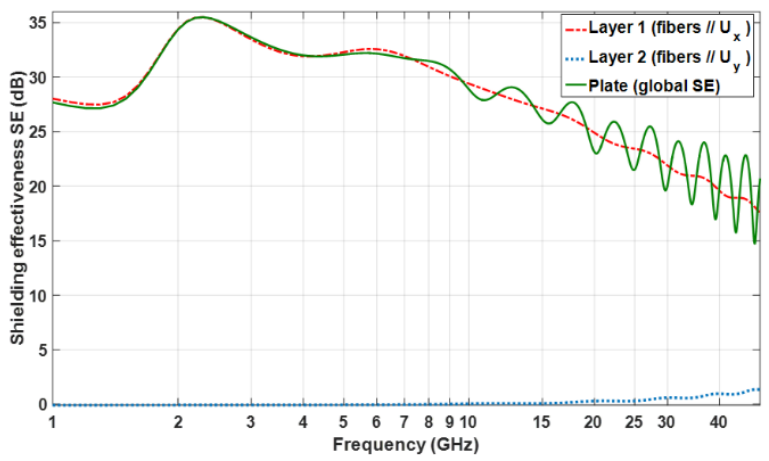

Fig. 6. Shielding effectiveness of a two layers composite plate with a $10^{3} \mathrm{~S} / \mathrm{m}$ contrast between the fibers and the matrix

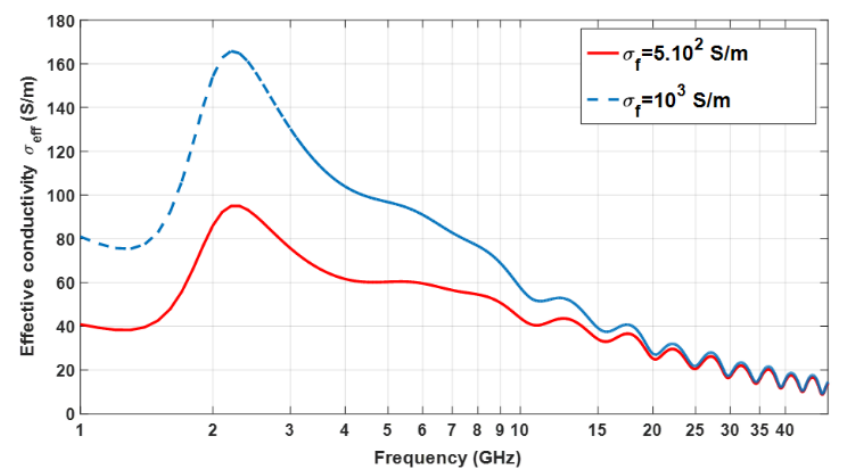

Fig. 7. Effective conductivities for two case studies with different contrasts in conductivity between the fibers and the matrices.

To homogenize the multilayer plate, the optimization algorithm in II-B is applied to two plates with different contrasts in conductivity. Fig. 7 shows that while the effect of having more conductive fibers is clear at low frequencies, the skin effect tends to minimize these differences as the frequency gets higher.

\section{CONCLUSION}

In this paper, a broadband homogenization method is proposed to extract the effective conductivity of composite plates.
This effective conductivity is the value which, applied to a homogeneous plate, gives the same transmission coefficient as that of the composite plate. The approach has been validated with cylindrical fibers. The effect of the direction of the fibers with respect to the incident field has also been studied. This work answers to the limits of classical quasi-static mixing rules that are restricted to composites with low volume fractions at relatively low frequencies. The results have also been compared to dynamic homogenization techniques recently proposed. Such homogenization techniques validated for planar shields are needed to evaluate the shielding effectiveness of enclosures with complex shapes made from composite materials and for which brute force 3D computations using full wave solvers is not possible. Woven composites will be the main challenge addressed in the future.

\section{APPENDIX}

\section{A. Dynamic homogenization method}

The tensor of effective complex permittivity $\varepsilon_{D H M}$ of a multiphasic composite material is calculated in [5] by adapting the homogenization technique with inclusion problems to dynamic conditions. This is done using the following averaging:

$$
\begin{aligned}
\varepsilon_{D H M}= & \left\langle\varepsilon^{i} \cdot\left(I+N^{i} \cdot \varepsilon^{\infty^{-1}} \cdot\left(\varepsilon^{i}-\varepsilon^{\infty}\right)\right)^{-1}\right\rangle \\
& \cdot\left\langle\left(I+N^{i} \cdot \varepsilon^{\infty^{-1}} \cdot\left(\varepsilon^{i}-\varepsilon^{\infty}\right)\right)^{-1}\right\rangle^{-1}
\end{aligned}
$$

where

$\varepsilon^{\mathrm{i}}$ is the permittivity of the phase I,

$N^{i}$ is the depolarization tensor of the phase I and

$\varepsilon^{\infty}$ the infinite medium permittivity.

\section{ACKNOWLEDGMENT}

This work is supported by the Automotive Mechatronics Chair, a cooperation between Faurecia, CentraleSupelec and Esigelec.

\section{REFERENCES}

[1] K. Bal, V. K. Kothari, "Permittivity of woven fabrics: A comparison of dielectric formulas for air-fiber mixtures”, IEEE Trans. on Dielectrics and Electrical Insulation. Vol. 17, pp. 881-889, June 2010.

[2] M. S. Mirotznik, S. Yarlagadda, R. McCauley, P. Pa, "Broadband electromagnetic modeling of woven fabric composites", IEEE Trans. on Microwave theory and techniques. Vol. 60, pp. 158-169, Jan. 2012.

[3] R. Tumayan, X. Bunlon, A. Reineix, "A method using an open TEM cell to extract the complex permittivity of an unknown material”, 2014 International Symposium on EMC, pp. 1102-1106, Oct. 2014.

[4] D. Micheli et al., "Electromagnetic characterization and shielding effectiveness of concrete composite reinforced with carbon nanotubes in the mobilephones frequency band”, Materials science and engineering $B$ 188, pp. 119-129, 2014.

[5] V. Préault, R. Corcolle, L. Daniel, L. Pichon, "Effective permittivity of shielding composite materials for microwave frequencies", IEEE Trans. on Electromagnetic Compatibility, Vol. 55, No. 6, pp. 1178-1186, 2013.

[6] V. Kelner, F. Capitanescu, O. Leonard, L. Wehenkel, "A hybrid optimization technique coupling an evolutionary and a local search algorithm", Journal of Computational and Applied Mathematics 215, pp. 448-456, 2008.

[7] Z. Michalewicz, Genetic Algorithms + Data Structures = Evolution Programs, 3rd ed. New York: Springer 1996, revised and extended edition.

[8] David M. Pozar, Microwave Engineering, $4^{\text {th }}$ edition Wiley, 2012. 